\title{
Inflammation and human cerebral aneurysms: current and future treatment prospects
}

\author{
Joseph S Hudson', Danielle S Hoyne² \& David M Hasan*3 \\ 'University of lowa, lowa City, IA, USA \\ 2Department of Otolaryngology, University of lowa Carver College of Medicine, lowa City, IA, USA \\ ${ }^{3}$ Department of Neurosurgery, University of lowa Hospitals \& Clinics, 200 Hawkins Drive, lowa City, \\ |A 52240, USA \\ *Author for correspondence: Tel.: +1 3193848669 m. Fax: +1 3193536606 m david-hasan@uiowa.edu
}

The formation of cerebral aneurysms and their rupture propensity is of immediate clinical importance. Current management includes observation with expectant management, microsurgical clipping and/or endovascular coiling. The surgical options are invasive and are not without increased risk despite the technological advances. Recent human and animal studies have shown that inflammation plays a critical role in aneurysm formation and progression to rupture. Modulating this inflammatory process may prove to be clinically significant. This review will discuss cerebral aneurysm pathogenesis with a focus on current and future research of potential use of pharmaceutical agents that attenuate inflammation in the aneurysm wall leading to decreased risk of aneurysm rupture.

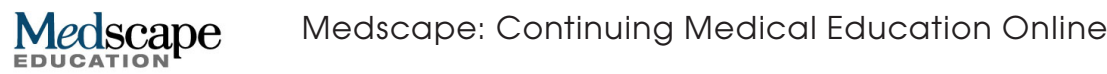

This activity has been planned and implemented in accordance with the Essential Areas and policies of the Accreditation Council for Continuing Medical Education through the joint sponsorship of Medscape, LLC and Future Medicine Ltd. Medscape, LLC is accredited by the ACCME to provide continuing medical education for physicians.

Medscape, LLC designates this journal-based CME activity for a maximum of 1 AMA PRA Category 1 Credit(s) ${ }^{\mathrm{TM}}$. Physicians should claim only the credit commensurate with the extent of their participation in the activity.

All other clinicians completing this activity will be issued a certificate of participation. To participate in this journal CME activity: (1) review the learning objectives and author disclosures; (2) study the education content; (3) take the post-test with a $70 \%$ minimum passing score and complete the evaluation at www.medscape.org/journal/fnl; (4) view/print certificate.

Release date: 28 October 2013; Expiration date: 28 October 2014

\section{Learning objectives}

Upon completion of this activity, participants should be able to:

- Describe the role of inflammation in the pathogenesis underlying intracranial aneurysm (IAs), based on a review

- Outline diagnostic imaging of inflammation in IAs

- Analyze potential clinical therapeutic interventions targeting inflammation of IAs

\section{Keywords \\ - cerebral aneurysm \\ - ferumoxytol = inflammation \\ - macrophages \\ * subarachnoid hemorrhage}

\section{Future $\%$ Medicine part of}




\section{Financial \& competing interests disclosure}

Editor: Elisa Manzotti, Publisher, Future Science Group. Disclosure: Elisa Manzotti has disclosed no relevant financial relationships.

CME author: Laurie Barclay, MD, is a freelance writer and reviewer, Medscape, LLC. Disclosure: Laurie Barclay has disclosed no relevant financial relationships.

Author \& credentials: Joseph S Hudson, University of Iowa, Iowa City, IA, USA. Disclosure: Joseph S Hudson has disclosed no relevant financial relationships.

Danielle S Hoyne, MD, Department of Otolaryngology, University of Iowa Carver College of Medicine, Iowa City, IA, USA. Disclosure: Danielle S Hoyne has disclosed no relevant financial relationships.

David M Hasan, MD, Department of Neurosurgery, University of Iowa Carver College of Medicine, Iowa City, IA, USA; and Department of Neurosurgery, University of Iowa Hospitals \& Clinics, 200 Hawkins Drive, Iowa City, IA 52240, USA. Disclosure: David M Hasan has disclosed no relevant financial relationships.

No writing assistance was utilized in the production of this manuscript.

Intracranial aneurysms (IAs) are common cerebrovascular pathologies, which typically form at the bifurcations of cerebral arteries. Rupture of IAs results in subarachnoid hemorrhage (SAH) and occasionally intraparenchymal hemorrhage. It is estimated that $2.3 \%$ of western populations have intracranial aneurysms [1]. Over 30,000 SAHs are diagnosed in the USA every year. SAHs caused by IA rupture comprises $2-5 \%$ of all strokes [1]. Approximately $12 \%$ of patients die before receiving medical attention [2]; $40 \%$ of hospitalized patients die within 1 month after the event; and more than a third of those who survive have major neurologic deficits [1-7]. Furthermore, persistent cognitive deficits are present in many patients. Risk factors for the development of IAs include: familial history of IA, autosomal dominant polycystic kidney disease, fibromuscular dysplasia, cigarette smoking, cerebral infection, traumatic and penetrating injuries, and female gender. The ISUIA study and the recent UCAS Japan study identify the additional factors of posterior circulation aneurysms (including posterior communicating artery aneurysms): aneurysms greater than $7 \mathrm{~mm}$, sentinel headache, new onset of cranial nerve deficits, hypertension, low BMI, and alcohol consumption of more than $150 \mathrm{~g} /$ week are associated with a higher risk of SAH [8-10]. Morbidity and mortality associated with SAH still remain high ( $65 \%)$ despite significant clinical advances in diagnosis and therapy [7]. Currently, the only treatment modalities available are microsurgical clipping and/or endovascular coiling. These are invasive despite the advances in technology. The desire to develop noninvasive medical treatment is appealing. Recent human and animal studies have shown that inflammation plays a key role in aneurysm formation and progression to rupture. This could provide promising targets for novel noninvasive pharmaceutical intervention. This article will highlight current IA clinical interventions and cerebral aneurysm pathogenesis in both animal and human studies. The 'Therapeutic targets' section will detail specific studies while paying special attention to future therapeutic and diagnostic possibilities pertaining to inflammation within the wall of cerebral aneurysms.

\section{Current treatment modalities}

Current management of unruptured intracranial aneurysms include: observation with expectant management (especially for aneurysms less than $7 \mathrm{~mm}$ ), microsurgical clipping, endovascular coiling, stent-assisted coiling, Onyx ${ }^{\circledR}$ liquid embolization (eV3, CA, USA) and, more recently, flow-diverting stents. Despite significant regional variation in the rates of clipping versus endovascular coiling, several studies and meta-analyses show endovascular coiling is associated with better clinical outcomes when compared with surgical clipping, especially in aneurysms that could be treated equally with both modalities [11-14]. Coils currently used are available as either bare platinum or biologically active coated coils. Several studies showed that the use of biologically active coated coils, such as hydrogel and polymer polyglycolic-lactic acid coils, has lead to lower recurrence rates following successful coiling $[15,16]$. Additional research revealed that use of simvastatin-coated coils accelerated aneurysm neck endothelialization and organization in rat models [17]. Another form of endovascular treatment is the use of liquid embolic materials, such as Onyx HD500, which have been particularly effective in treating small IA with minimal associated risks in experienced hands [18]. The development of intracranial stents to assist in coiling significantly improved the treatment of wide-necked aneurysms. Navigation of the microcatheter through a pore in the stent or entrapment of the catheter between the vessel wall and the stent improves packing 
density, prevents coil protrusion into the parent artery, and diverts flow to allow vessel remodeling [19]. Advancement in stent design lead to the development of flow-diverting stents; the alteration in the dynamic fluid environment within the aneurysm is associated with high rates of complete occlusion [20-22]. The narrow mesh system leads to the reconstruction of the vessel wall and is ideal for treatment of fusiform, giant and/or wide-necked aneurysms [21]. Treatment of recurrent aneurysms following previous embolization or surgery is challenging. Chalouhi et al. recently reported that patients with recurrent aneurysms treated with the Pipeline Embolization Device ${ }^{\mathrm{TM}}$ (eV3) had an approximate occlusion rate of $93 \%$ [23]. While promising, endovascular treatment of IAs with flow diverters is relatively new, with only a small number of clinical results available to evaluate treatment efficacy. All of the aforementioned interventions have procedural complication rates which are far from negligible and, thus, illustrate the need for noninvasive alternatives and a more complete understanding of IA pathology.

\section{Inflammation \& cerebral aneurysms \\ Aneurysm formation \\ Hemodynamic stress, endothelial \\ dysfunction \& atherosclerosis}

Formation of IAs involves a combination of hemodynamic stress and inflammation. A delicate homeostatic balance exists between the relentless pulsating hemodynamic sheer stress of diastole-systole and vessel wall integrity. It is hypothesized that IAs form as a result of disturbances of this delicate balance [24,25]. During IA formation, the highly specialized structure of the vessel wall is transformed into a disorganized compilation containing fewer distinct layers of specialized tissue. Disruption of the internal elastic lamina at vessel bifurcations initiates IA formation [26]. Statistical characterization of the differential hemodynamic microenvironments at various cerebral vessel bifurcations revealed a high association between wall shear stress magnitude and aneurysm initiation [27]. This hemodynamic force is responsible for changes in endothelial localization, structure, morphology, and alignment. Sheer stress may also modulate intrinsic endothelial signaling. Aoki et al. showed hemodynamic force activated the proinflammatory pathway $\mathrm{PGE}_{2}-\mathrm{EP}_{2}$, which subsequently amplified chronic inflammation in a NF- $\mathrm{KB}$ dependent manner leading to increased expression of MCP-1, ICAM-1, VCAM-1, E-selectin, P-selectin, IL-6, TNF- $\alpha$ and IL-1 $\beta$ [28-33]. These cytokines lead to recruitment of inflammatory cells and initiation of the inflammatory process within the wall of cerebral aneurysms [24,25].

The role of atherosclerosis in the pathophysiology of cerebral aneurysms has been debated for decades. Based on classification of atherosclerosis by histological changes, nearly all cerebral aneurysms may be considered atherosclerotic. Features of advanced atherosclerosis (including a core of atheromatous debris, a fibrous cap, macrophages and $T$ cells) are observed in approximately half of IAs; myointimal hyperplasia is reported to occur with a similar incidence. Thrombosis occurs in some cerebral aneurysms, which may further amplify the inflammatory reaction and wall degeneration, with a loss of tensile strength and, ultimately, rupture [34-36].

\section{Vascular smooth muscle cell phenotypic modulation}

Hemodynamic stress, in addition to inflammatory cytokines, produced by activated endothelial cells and recruited inflammatory cells mediates phenotypic modulation of vascular smooth muscle cells (VSMCs), principally contractile in nature to the proinflammatory and dedifferentiated synthetic phenotype. These modulated cells are characterized by their lack of contractile protein production as shown by Nakajima et al. [37]. Immunohistochemical staining utilizing antibodies to $\alpha$-smooth muscle actin, desmin and smooth muscle myosin heavychain isoforms clearly illustrated how VSMCs in control arteries demonstrate increased expression of contractile proteins compared with VSMCs in unruptured aneurysm walls [37]. VSMCs in experimentally induced IAs also display decreased collagen I and II biosynthesis at the transcriptional level through proinflammatory NF- $\mathrm{KB}$ transcription factor and cytokine IL-1 $\beta$ activity [30]. Other phenotypically indicative differential expression in VSMCs include proinflammatory transcription factor Ets-1, matrix metalloproteinases (MMPs) whose catalytic activity degrades the extracellular matrix and $\mathrm{p} 47 \mathrm{phox}$ (a subunit of NADPH oxidase) [38-40]. Apoptosis and subsequent reductions in the medial density of VSMCs are also highly associated with IA pathogenesis [41,42]. Indeed, capase-3 expression (critical in the apoptotic cascade) has been implicated in the pathogenesis of IAs using real-time PCR in VSMC samples from human IAs [42].

In addition to IA formation, Nakajima et al. showed VSMCs in ruptured aneurysms appeared to have lost both phenotypes (characterized by 
further decreases in contractile protein staining intensity), thus suggesting phenotypic modulation of VSMCs plays a role in aneurysm rupture [37]. In summary, as the aneurysm wall becomes sufficiently fragile as a result of cell death and increased matrix turnover, it will succumb to intraluminal pressure and rupture. Thus, the modulation of VSMCs to the synthetic and proinflammatory phenotype is an integral constituent of both IA pathogenesis and rupture progression.

\section{Inflammatory mediators}

Leukocyte invasion is commonly observed during the progression of IAs. Specifically, macrophage accumulation within the walls of IAs is well documented [43,44]. Macrophage-derived extracellular matrix remodeling was shown to occur partially by MMP-2 and MMP-9 secretion in a rat model of experimentally induced IA [45]. In addition, studies have investigated the levels of MCP-1, a macrophage-attracting cytokine, in both humans and animal models of IA. MCP-1 knockout mice showed decreased IA formation, lower levels of macrophage recruitment, and inhibited MMP-2 and MMP-9 expression [29]. A recent study also detailed the patient characteristic-specific expression levels of two other cytokines, IL-1 $\beta$ and TNF- $\alpha$. All three regulatory cytokines were expressed at increased levels when compared with controls. Patients with more than one aneurysm displayed higher levels of both MCP- 1 and TNF- $\alpha$ than patients with just one [46]. Furthermore, Morgan et al. illustrated how functional single nucleotide promoter polymorphisms at -174 and -572 (which correlated to increased synthesis of IL-6) were statistically correlated with increased IA susceptibility [47]. Since the initial correlation, several other studies were conducted to elucidate a potential allelic association in various population cohorts $[31,48]$. Ultimately, a meta-analysis revealed that the G572C polymorphism showed a statistically significant association with ruptured and unruptured aneurysms. Interestingly, the G174C polymorphism was shown to have a statistically significant protective effect against IA [49]. These studies further exemplify the importance of proinflammatory cytokines and ultimately suggest a potential allelic basis for IA formation pathophysiology. In addition to chemoattractant and proinflammatory cytokines, the NF- $\kappa \mathrm{B}$ transcription factor is a mediator of macrophage recruitment to the aneurysm wall. Aoki et al. showed NF- $\kappa B$ was overexpressed in the IA wall [50]. Costaining of the activated DNA binding form of NF- $\kappa B$ with MCP-1 and VCAM-1, both of which are macrophage localization prerequisites, suggested a molecular basis through which NF- $\kappa \mathrm{B}$ activations recruits macrophages during IA inflammation [50]. To further elucidate NF- $\kappa B$ activation during IA formation, the authors created mice deficient in the $\mathrm{p} 50$ subunit of NF- $\mathrm{KB}$ in their previously established experimentally induced IA model. They found reductions in macrophage recruitment, MCP- 1 and VCAM-1 mRNA, aneurysmal changes such as tears in the internal elastic lamina and overall IA size. Additionally, $\mathrm{p}^{5-} \mathrm{S}^{-}$mice showed no upregulation of MMP-2, MMP-9, IL-1 $\beta$ and inducible nitric oxide synthetase (iNOS; induces apoptosis in medial smooth muscle cells) mRNA [51]. These findings suggest NF- $\kappa \mathrm{B}$ is a key mediator in the formation of IA. Mast cells are also leukocytes involved in inflammatory responses. Mast cells typically act through the release of various granulized cytokines. Recently, their role in IA formation was accessed in an experimentally induced model of IA in rats. Ishibashi et al. found an increased number of mast cells in IA walls during the formation of IA [52]. They also found in cultured rat VSMCs, induction of MMPs after mast cell degranulation, suggesting a potential role for mast cells in IA pathogenesis [52]. Immunoglobulins and complement have also been detected in IA walls. Chyatte $e t$ al. found that tissue from human aneurysm wall collected during microsurgical clipping is rich with C3a, along with VCAM-1, macrophages and $\mathrm{T}$ lymphocytes [53].

\section{Aneurysm rupture}

Inflammatory cell invasion is also associated with IA rupture. Recently, Hasan et al.further investigated the role of mast cells and macrophage imbalance in human tissue samples [54]. Macrophages in atherosclerotic plaques can be divided into two major subtypes: $\mathrm{CD} 14^{\text {high }} \mathrm{CD} 16^{\text {low }}$ and $\mathrm{CD} 14^{\text {low }} \mathrm{CD} 16^{\text {high }}$. These two subtypes were found to play opposing roles during inflammation [55-57]. In unruptured IA, immunostaining of the surgically resected aneurysmal dome tissue revealed pro- and anti-inflammatory subset macrophages were in balance with few mast cells present. Comparatively, in ruptured aneurysm walls, there was a deviation from the critical macrophage balance. That is, proinflammatory macrophage expression far exceeded anti-inflammatory macrophage expression. In addition, the authors observed significant upregulation of mast cells. They hypothesized that this polarized proinflammatory immune response coupled 
with an increased mast cell presence may contribute to the cascade of molecular events that trigger IA rupture. However, it was unclear if the increased expression and polarization occurred following IA rupture, or if it proceeded rupture [54]. T cells also participate in the inflammatory reaction in conjunction with macrophages. In fact, T-cell and macrophage localization in the IA wall is associated with an increased propensity to rupture [44]. It is also unclear whether these molecular changes were present in IA before rupture or occur as a response to aneurysm rupture. C3a and C5a were further implicated in the pathogenesis of ruptured aneurysms [58]. Immunostaining for complement activation (membrane attack complex) in human surgically resected fundi revealed consistent localization in the decellularized region in the IA outer wall. In addition, the proportionality of membrane attack complex positive area in relation to total IA surface area was greater in ruptured IA versus unruptured [58]. Further research by the same group elucidated that the outer IA wall lacked complement inhibitor protection, that is, complement inhibitor factor $\mathrm{H}$, and complement inhibitor-binding glycosaminoglycans [59]. This was hypothesized to subject the outer wall to the full blown complement response.

The aforementioned research studies in humans and animals suggest that the mechanism of cerebral aneurysm formation and rupture are different. The goal is that continued understanding of the pathogenesis of aneurysmal formation and rupture would lead to the discovery of potential therapeutic agents that could interrupt this process, achieving a steady state where aneurysms become stable in size and shape without progression to rupture and the necessity of surgical intervention.

\section{Diagnostic imaging}

Collectively, all the studies above have shown that inflammation plays an integral part of aneurysm formation and progression to rupture $[24-28,30,44,50,52-54,58,59]$. The development of molecular and cellular imaging to monitor inflammation amelioration or deterioration in vivo will allow clinicians to better predict the probability of IA rupture. An animal study by Deleo et al. developed a method to access the inflammation through clinical field strength MRI of myeloperoxidase (MPO), an inflammatory enzymatic biomarker responsible for MMP activation and reactive oxygen species creation [60]. Experimentally induced IAs in rabbits were imaged using a MPO-specific magnetic resonance contrast agent di-5-hydroxytryptamide of gadopentetate dimeglumine, which is a paramagnetic MPO substrate. Imaging revealed a difference in enhancement ratio between control aneurysms and inflamed aneurysms injected with Escherichia coli lipopolysaccharide, which were confirmed to have greater tissue-specific MPO expression using immunohistochemistry [60]. The findings of this study suggest that inflammatory enzyme-specific imaging may aid in the identification of patients harboring IAs, with a propensity to rupture.

Recently, Hasan and colleagues used novel cellular imaging of macrophages as a surrogate biomarker for inflammation [61]. Human patients with cerebral aneurysms were infused with ferumoxytol and subsequently imaged using a special T2*-MRI sequence. As ferumoxytol is cleared by reticuloendothelial system macrophages, the macrophages localized within the wall of cerebral aneurysms were visualized using this technique and then their presence further confirmed by immunohistological staining. Further studies suggest that this technique could be valuable in predicting which aneurysm is at increased risk and warrants urgent treatment [61-63]. Aneurysms with early ferumoxytol uptake (24 h), showed increased expression of inflammatory molecules and cells when compared with later uptake. These results suggest early in vivo macrophage uptake of ferumoxytol points to aneurysmal instability [61,62]. Furthermore, an optimal dosage protocol and imaging chronology $(5 \mathrm{mg} / \mathrm{kg}$ of ferumoxy tol with imaging at 0 and $72 \mathrm{~h}$ postinfusion) were established [63]. Such radiologic imaging techniques will probably play essential roles in the future clinical assessment and management of IA. The group was also able to use this technique to monitor the therapeutic effect of aspirin in attenuating the inflammatory process within the wall of human cerebral aneurysms [64].

\section{Potential therapeutic targets Animal model}

Establishing experimentally induced IA in animal model systems allows for the elucidation of mechanistic underpinnings and progressive observation of IA not possible in humans. Many mechanistic discoveries pertaining to IA formation and progression have been made utilizing animals with artificially increased hemodynamic stress with and without elastase infusion [65]. The most frequent models utilized in the past include the Hashimoto model of cerebral aneurysms in rodents and surgical construction of cerebral 
aneurysm pouch in combination with elastase infusion. These two models are beneficial in evaluating the mechanistic pathways of aneurysm formation, but not rupture, as both fail to progress to rupture.

The rupture of IAs is of immediate clinical importance; thus, animal models with a high frequency of spontaneous rupture are required. Such a model was developed in hypertensive mice by Nuki et al. [66]. Stereotaxic injection of elastase into the basal cistern resulted in formation of large IA (three-to-five-times the size of the parent artery), and subsequent aneurysm rupture and development of SAH [66]. This model and other future animal models with spontaneous rupture propensity will aid in the development of molecularly specific preventative pharmacological therapeutics.

\section{Therapeutic targets}

As previously noted, the NF- $\kappa \mathrm{B}$ is a key transcription factor responsible for the regulation of inflammatory genes during IA formation. Aoki et al. used a synthesized decoy oligodeoxynucleotide (ODN) to bind NF- $\mathrm{KB}$ and consequentially inhibit NF- $\kappa \mathrm{B}$ DNA binding in rats $[50,67]$. Incidence of aneurysmal changes, disruption of the internal elastic lamina, and aneurysm size were all significantly reduced when ODNs were administered 1 week after aneurysmal induction. Likewise, ODN administration reduced macrophage recruitment to the aneurysm wall through reduction of VCAM-1 and MCP-1 [50]. Another previously described transcription factor, ETS-1, is also involved in transcriptional regulation of inflammation [40]. A recent study by this group suggests a simultaneous administration of chimeric ODNs for both NF- $\kappa B$ and ETS-1 synergistically acts to repress molecular mediators of inflammation while rescuing collagen biosynthesis. The authors speculate that pharmaceutical administration of chimeric decoy ODNs may be able to regress IA [32]. However, the authors noted that if ODNs are administered transorally or transvenously, it is degraded by nucleases within a few hours. In the aforementioned experiment the decoy ODN was administered intrathecally. Miyake et al. developed an ODN structural modification that increased nuclease resistance and was able to attenuate abdominal aortic aneurysms after intraperitoneal administration in a rat model [68]. In the future, when nuclease-resistant ODNs are readily available, systemic administration targeting ETS- 1 and NF- $\kappa B$ transcription factors may prove clinically efficacious.
Statins have various protective effects on vascular disease, thus Aoki et al. further investigated their therapeutic potential in a rat-induced IA model [69,70]. To examine whether or not simvastatin had a suppressive effect on pre-existing aneurysms, oral administration began 1 month after aneurysm induction. Immunohistochemical analysis revealed decreased expression of IL-1 $\beta$, iNOS, MMP-2 and MMP-9 along with concomitant decreases in macrophage recruitment [69]. Interestingly, Tada et al. found lower doses of pravastatin attenuated IA progression while high doses promoted aneurysm growth and rupture through activation of apoptotic capase-3 and dUTP nick-end labeling (TUNEL)-positive cells. Thus, current evidence suggests that statins may exert dose-dependent bidirectional effects on aneurysm pathogenesis [70]. Furthermore, a recent hospital-based case-control analysis of statin use concluded that an inverse relationship exists between statin use and IA rupture [71]. Further research is needed to assess the clinical efficacy of statin administration pertaining to IA.

Aoki et al. investigated the effects of selective MMP-2 and MMP-9 inhibitions (tolylsam) on aneurysm progression. After administration, the progression of IA was inhibited, suggesting an inhibition of MMP gelatinase activity. Since the total number of aneurysmal changes was not different between the tolylsam administered rats and control rats, the authors suggested tolylsam may have only delayed the progression of IA [45]. The therapeutic potential of MCP-1 was explored by Aoki et al. with targeted gene therapy utilizing a dominant negative MCP-1 DNA molecule (7ND) [29]. Cells translated and secreted the 7ND protein into the blood stream, which subsequently bound to MCP-1 receptors on target monocytes. The aforementioned therapy reduced macrophage recruitment and subsequent MMP-2, MMP-9 and iNOS induction. In addition, the authors observed a significant decrease in aneurysm size [29]. Thus, based on several gene therapy studies, MCP-1 may be an ideal target for therapeutic treatment of IA [29,33,72].

Ishibashi et al. observed an increased number of mast cells present in the IA wall [52]. Furthermore the authors documented mast cell induced upregulation of MMP-2, MMP-9 and iNOS following degranulation and subsequent release of proinflammatory cytokines. Treatment of an experimentally induced IA rat model with mast cell degranulation inhibitors tranilast and emedastine resulted in a decrease in aneurysm size and 
an increase in media thickness [52]. Since mast cell inhibitors are widely used as antiallergenic drugs, the safety and efficacy are well established. Thus, mast cell inhibitors may be ideal for pharmaceutical attenuation of inflammatory mechanisms pertaining to IA degradation.

Cathepsins are lysosomal proteolytic enzymes that degrade unnecessary intracellular or endocytosed proteins [73]. Cathepsins also display collagenolytic activity outside of the lysosome or endosome [74]. Aoki et al. showed increased cathepsins B, K, S (classified by catalytic site) expression in both rat-induced IA and human IA tissue suggesting a mechanistic role for cathepsins in vascular remodeling and IA degradation. Consistent with MMP-2 and MMP-9 inhibition, selective inhibition of cathepsins (utilizing orally administered cysteine cathepsin inhibitor NC-2300 immediately following experimental IA induction) yielded significant reductions in late-stage aneurysm progression. That is, extracellular matrix remodeling activity of cystine cathepsins was inhibited. There were no changes in macrophage infiltration or early aneurysmal changes between the treatment group and the control group [75]. Thus, selective inhibitors of cathepsin-mediated extracellular matrix remodeling may be ideal therapeutic targets in the prevention of IA progression.

The aforementioned macrophage expressing reactive oxygen species producing gene and subsequent reactive oxygen species were shown to be critical in IA formation [51]. Aoki et al. furthered their hypothesis by orally administering edaravone (Mitsubishi Tanabe Pharma Corporation, Tokyo, Japan), a free radical scavenger, to an experimentally induced model of IA in rats [39]. The size of induced IA was significantly smaller in the group treated with edaravone. Likewise, medial degradation was inhibited in the treatment group without influencing systemic blood pressure. Also, edaravone inhibited NF- $\kappa \mathrm{B}$ activation through reactive oxygen species elimination, thus resulting in decreased MCP-1, MMP-2 and VCAM-1. As edaravone is widely used in the treatment of patients with acute cerebral infarction, edaravone is one of the leading candidates for molecular treatment of IA through the elimination of reactive oxygen species [76,77].

Yagi et al. examined the proinflammatory role of PDE-4, whose catalytic activity hydrolyzes cyclic AMP, and is involved in diseases of inflammation $[78,79]$. They demonstrated that PDE-4 was activated in an induced rat IA model and was associated with increased MMP and macrophage migration. The authors then orally administered a PDE-4 inhibitor (ibudilast) and observed an inhibition of formation and progression of IA along with concomitant downregulation of inflammatory molecules. However, ibudilast possesses antiplatelet effects; thus, the authors recommend that ibudilast not be administered prior to endovascular or microsurgical treatment of ruptured IA. Ibudilast administration may prove to be beneficial for patients with IA requiring antithrombotic therapy [79].

Recently, Ishibashi et al. showed intraperitoneally administered imidapril, an angiotensinconverting enzyme inhibitor (the key enzyme in the inflammatory renin-angiotensin system) and potent MMP-9 inhibitor reduced the size and medial thinning of experimentally induced aneurysms in rats [80]. Immunostaining and western blot analysis revealed imidapril did not influence angiotensin-converting enzyme expression in aneurysm walls. Further western blotting indicated that MMP-9 levels were similar in control arteries when compared with IA tissue. However, assays of gelatinolytic activity postimidapril administration revealed reductions in MMP-9 catalysis in aneurysm tissue [80]. These findings suggest imidapril may prove to be efficacious in targeted IA treatment through selective inhibition of MMP-9.

Finally, Hasan et al. performed a secondary analysis of the ISUIA study and showed that frequent aspirin use leads to decreased risk of aneurysm rupture by $60 \%$ [64]. The same groups then sought to determine the direct effects of acetylsalicylic acid (asprin) on inflammatory molecules and cells within the wall of human cerebal aneurysms using radiological and histological techniques [81,82]. Their preliminary data suggest that a low dose of acetylsalicylic acid (81 mg daily for 3 months) may attenuate inflammation in IA. Histologically, a decrease in macrophage and proinflammatory molecules COX-2 and mPGES-1 expression was observed in aspirin-treated patients [81]. These molecules were found to be expressed more frequently in ruptured aneurysms [82]. The potential beneficial effects of aspirin must be weighed against its antiplatelet affects in the setting of acute SAH. However, a recent correlative study of aspirin use was associated with a lower rate of hemorrhagic presentation with no adverse effects on presenting clinical grade, vasospasm or outcome concurrently observed [83]. These findings suggest low doses of aspirin may be beneficial in the administration of treatment to patients with IA and SAH. 


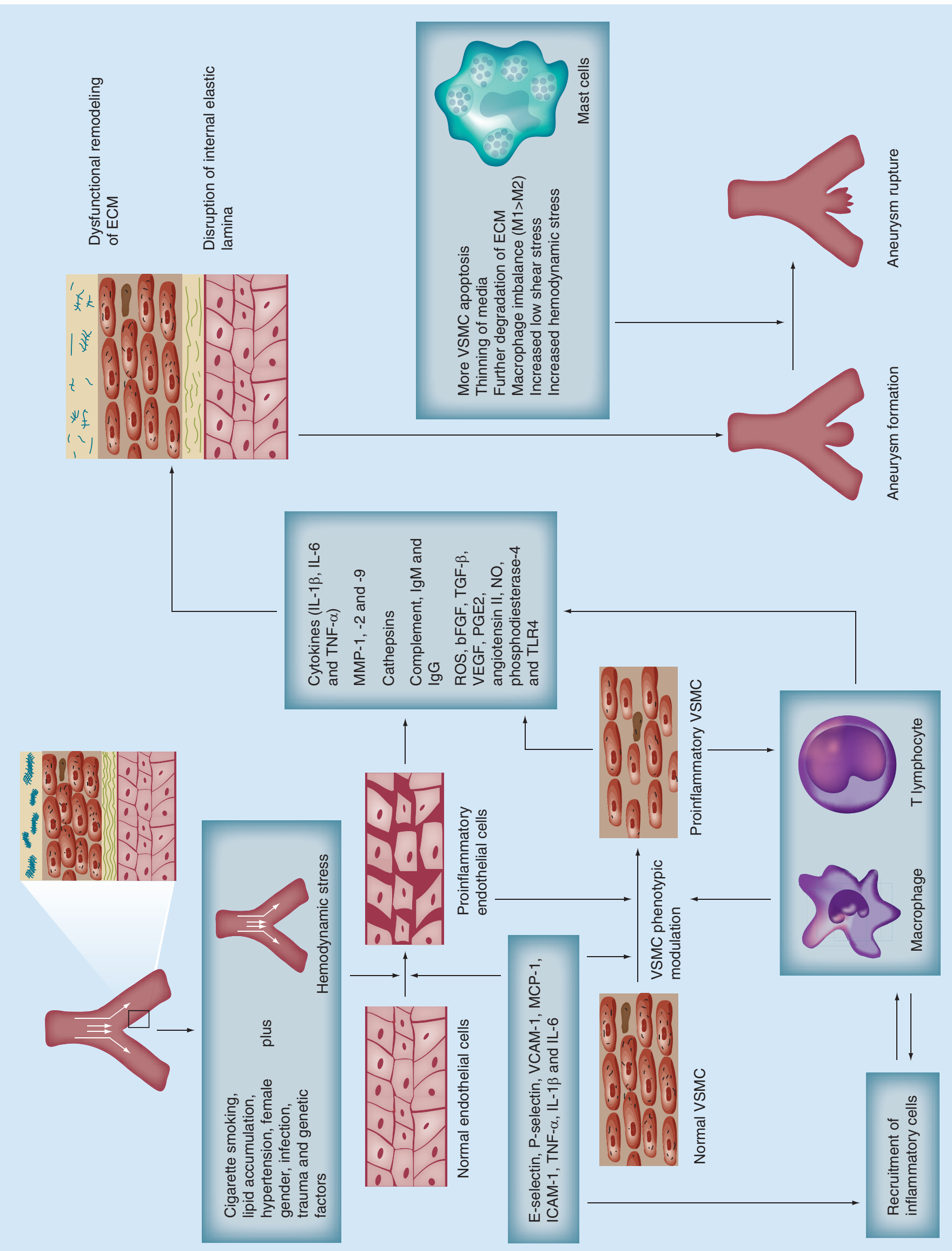


Figure 1. Summary of the interaction among environmental factors, genetic preconditioning, hemodynamic stress and inflammation in aneurysm formation and progression to rupture (facing page).

ECM: Extracellular matrix; M1: Proinflammatory macrophage; M2: Anti-inflammatory macrophage; NO: Nitric oxide; ROS: Reactive oxygen species; VSMC: Vascular smooth muscle cell.

\section{Conclusion}

Recent human and animal studies have shown that inflammation plays a key role in aneurysm formation and progression to rupture (FIGURE 1). Ongoing research and preliminary data show the possibility of modulating this inflammatory process in the aneurysm wall. Furthermore, these molecular interventions may potentially halt this deleterious process. Likewise, techniques that allow for inflammatory imaging in vivo will greatly improve clinical assessment of IAs. Used in conjunction, these notable medical advances create an alternative noninvasive medical treatment for the management of cerebral aneurysms.

\section{Future perspective}

Advances in microsurgical and endovascular technology will inevitably lead to lower rates of complication and improve respective patient outcomes. However, as the etiological unraveling of IA pathogenesis continues, our ability to develop effective, systemic, transorally administered pharmaceuticals will follow suit. Equally important is the development of molecularly based imaging of IA progression. Further investigation into the complex inflammatory mechanisms underlying cerebral aneurysm formation will usher in a new era of clinical competency and preferable patient outcomes.

\section{Executive summary}

\section{Intracranial aneurysms: current treatment modalities}

- Relatively invasive, surgical procedures are implemented to treat intracranial aneurysms (IAs) include: neurosurgical clipping, endovascular coil embolization, stent-assisted coiling, liquid embolization and flow-diverting stents.

\section{Endothelial cell modulation}

- Increased hemodynamic stress coupled with development of atherosclerotic changes leads to increased expression of E- and P-selectins, MCP-1, VCAM-1 and ICAM-1 resulting in recruitment of inflammatory cells and infiltration of the subendothelial layers.

\section{Vascular smooth muscle cell phenotypic modulation}

- Progressive phenotypic differentiation of vascular smooth muscle cells to a synthetic and proinflammatory state is essential for IA pathogenesis. This is exemplified by experimentally observed differential expression of contractile proteins, NF- $\kappa \mathrm{B}, \mathrm{matrix}$ metalloproteinases (MMPs), caspase-3, ETS-1 and p47phox.

\section{Inflammatory mediators}

- IA tissue contains many inflammatory effectors, such as macrophages, MMP-2-9, MCP-1, IL-1 $\beta$, TNF- $\alpha$, NF- $\kappa B$, VCAM-1, mast cells, complement and immunoglobin. In addition, there is differential production of reactive oxygen species.

\section{Diagnostic imaging of inflammation}

- The role of inflammation in cerebral aneurysm pathogenesis can be visualized in vivo. This provides important clinical information, and will greatly improve IA management. Macrophage (ferumoxytol) and myeloperoxidase (di-5-hydroxytryptamide of gadopentetate dimeglumine)-specific inflammatory imaging are current experimentally efficacious methodologies.

\section{Targeted therapies}

- Research has revealed potential targets for molecular treatment of IA. Potential treatments include oligodeoxynucleotide administration, MMP and MCP-1 inhibition, cathespin inhibition, free radical scavengers, PDE-4 inhibition and aspirin administration.

\section{Conclusion}

- The role of inflammation in IA formation and progression has been well established. Noninvasive molecularly targeted clinical interventions will play an important role in future IA management.

\section{References}

Papers of special note have been highlighted as:

- of interest

-" of considerable interest

1. Wiebers DO, Whisnant JP, Sundt TM Jr, O'Fallon WM. The significance of unruptured intracranial saccular aneurysms. J. Neurosurg. 66(1), 23-29 (1987).
2. Wiebers DO, Whisnant JP, O'Fallon WM. The natural history of unruptured intracranial aneurysms. N. Engl. J. Med. 304(12), 696-698 (1981).

3. Vlak MH, Algra A, Brandenburg R, Rinkel GJ. Prevalence of unruptured intracranial aneurysms, with emphasis on sex, age, comorbidity, country, and time period: a systematic review and meta-analysis. Lancet Neurol. 10(7), 626-636 (2011).

4. Juvela S. Prevalence of and risk factors for intracranial aneurysms. Lancet Neurol. 10(7), 595-597 (2011).

5. Wermer MJ, Van Der Schaaf IC, Algra A, Rinkel GJ. Risk of rupture of unruptured intracranial aneurysms in relation to patient 
and aneurysm characteristics: an updated meta-analysis. Stroke 38(4), 1404-1410 (2007).

6. Woo D, Khoury J, Haverbusch MM et al. Smoking and family history and risk of aneurysmal subarachnoid hemorrhage. Neurology 72(1), 69-72 (2009).

7. Wiebers DO, Whisnant JP, Huston J 3rd et al. Unruptured intracranial aneurysms: natural history, clinical outcome, and risks of surgical and endovascular treatment. Lancet 362(9378), 103-110 (2003).

8. Clarke M. Systematic review of reviews of risk factors for intracranial aneurysms. Neuroradiology 50(8), 653-664 (2008).

9. Feigin VL, Rinkel GJ, Lawes CM et al. Risk factors for subarachnoid hemorrhage: an updated systematic review of epidemiological studies. Stroke 36(12), 2773-2780 (2005).

10. Investigators UJ, Morita A, Kirino $\mathrm{T}$ et al. The natural course of unruptured cerebral aneurysms in a Japanese cohort. N. Engl. J. Med. 366(26), 2474-2482 (2012).

11. Hwang JS, Hyun MK, Lee HJ et al. Endovascular coiling versus neurosurgical clipping in patients with unruptured intracranial aneurysm: a systematic review. BMC Neurol. 12, 99 (2012).

12. Lanzino G, Murad MH, D’urso PI, Rabinstein AA. Coil embolization versus clipping for ruptured intracranial aneurysms: a meta-analysis of prospective controlled published studies. AJNR Am. J. Neuroradiol. 34, 1764-1768 (2013).

13. Health Quality O. Coil embolization for intracranial aneurysms: an evidence-based analysis. Ont. Health Technol. Assess. Ser. 6(1), 1-114 (2006).

14. Molyneux AJ, Kerr RS, Yu LM et al. International subarachnoid aneurysm trial (ISAT) of neurosurgical clipping versus endovascular coiling in 2143 patients with ruptured intracranial aneurysms: a randomised comparison of effects on survival, dependency, seizures, rebleeding, subgroups, and aneurysm occlusion. Lancet 366(9488), 809-817 (2005).

15. Ansari SA, Dueweke EJ, Kanaan Y et al. Embolization of intracranial aneurysms with second-generation matrix-2 detachable coils: mid-term and long-term results. J. Neurointerv. Surg. 3(4), 324-330 (2011).

16. Reinges MH, Krings T, Drexler AY et al. Bare, bio-active and hydrogel-coated coils for endovascular treatment of experimentally induced aneurysms. Longterm histological and scanning electron microscopy results. Interv. Neuroradiol. 16(2), 139-150 (2010).
17. Kodama T, Iwata H. Comparison of bare metal and statin-coated coils on rates of intra-aneurysmal tissue organization in a rat model of aneurysm. J. Biomed. Mater. Res. B Appl. Biomater. 101(4), 656-662 (2013).

18. Simon S, Archer K, Mericle R. Multicenter registry of liquid embolic treatment of cerebral aneurysms. World Neurosurg. doi:10.1016/j.wneu.2013.03.070 (2013) (Epub ahead of print).

19. Seibert B, Tummala RP, Chow R, Faridar A, Mousavi SA, Divani AA. Intracranial aneurysms: review of current treatment options and outcomes. Front. Neurol. 2, 45 (2011).

20. Simgen A, Junk D, Reith W. [Flow diverter: a new therapy option for intracranial aneurysms]. Radiologe 52(12), 1118-1124 (2012).

21. Brinjikji W, Murad MH, Lanzino G, Cloft HJ, Kallmes DF. Endovascular treatment of intracranial aneurysms with flow diverters: a meta-analysis. Stroke 44(2), 442-447 (2013).

22. Roszelle BN, Gonzalez LF, Babiker MH, Ryan J, Albuquerque FC, Frakes DH. Flow diverter effect on cerebral aneurysm hemodynamics: an in vitro comparison of telescoping stents and the pipeline. Neuroradiology 55(6), 751-758 (2013).

23. Chalouhi N, Chitale R, Starke RM et al. Treatment of recurrent intracranial aneurysms with the Pipeline Embolization Device. J. Neurointerv. Surg. doi:10.1136/ neurintsurg-2012-010612 (2013) (Epub ahead of print).

24. Chalouhi N, Ali MS, Jabbour PM et al. Biology of intracranial aneurysms: role of inflammation. J. Cereb. Blood Flow Metab. 32(9), 1659-1676 (2012).

-. Review of intracranial aneurysm (IA) pathogenesis and summary of therapies targeting inflammation in the IA wall.

25. Tulamo R, Frosen J, Hernesniemi J, Niemela M. Inflammatory changes in the aneurysm wall: a review. J. Neurointerv. Surg. 2(2), 120-130 (2010).

- Review of the literature pertaining to inflammation in the IA wall and degenerative mechanisms leading to IA rupture.

26. Hashimoto N, Handa H, Nagata I, Hazama F. Animal model of cerebral aneurysms: pathology and pathogenesis of induced cerebral aneurysms in rats. Neurol. Res. 6(1-2), 33-40 (1984).

27. Alfano J, Kolega J, Natarajan K et al. Intracranial aneurysms occur more frequently at bifurcation sites that typically experience higher hemodynamic stresses. Neurosurgery 73(3), 497-505 (2013).
28. Aoki T, Nishimura M, Matsuoka T et al. $\mathrm{PGE}_{2}-\mathrm{EP}_{2}$ signalling in endothelium is activated by haemodynamic stress and induces cerebral aneurysm through an amplifying loop via NF-кB. Br. J. Pharmacol. 163(6), 1237-1249 (2011).

29. Aoki T, Kataoka H, Ishibashi R, Nozaki K, Egashira K, Hashimoto N. Impact of monocyte chemoattractant protein-1 deficiency on cerebral aneurysm formation. Stroke 40(3), 942-951 (2009).

30. Aoki T, Kataoka H, Ishibashi R, Nozaki K, Morishita R, Hashimoto N. Reduced collagen biosynthesis is the hallmark of cerebral aneurysm: contribution of interleukin- $1 \beta$ and nuclear factor- $\kappa \mathrm{B}$. Arterioscler. Thromb. Vasc. Biol. 29(7), 1080-1086 (2009).

31. Zhang G, Tu Y, Feng W, Huang L, Li M, Qi S. Association of interleukin-6-572G/C gene polymorphisms in the Cantonese population with intracranial aneurysms. J. Neurol. Sci. 306(1-2), 94-97 (2011).

32. Aoki T, Kataoka H, Nishimura M, Ishibashi R, Morishita R, Miyamoto S. Regression of intracranial aneurysms by simultaneous inhibition of nuclear factor- $\mathrm{KB}$ and Ets with chimeric decoy oligodeoxynucleotide treatment. Neurosurgery 70(6), 1534-1543; discussion 1543 (2012).

33. Egashira K, Koyanagi M, Kitamoto S et al. Anti-monocyte chemoattractant protein-1 gene therapy inhibits vascular remodeling in rats: blockade of MCP-1 activity after intramuscular transfer of a mutant gene inhibits vascular remodeling induced by chronic blockade of NO synthesis. FASEB J. 14(13), 1974-1978 (2000).

34. Killer-Oberpfalzer M, Aichholzer M, Weis S et al. Histological analysis of clipped human intracranial aneurysms and parent arteries with short-term follow-up. Cardiovasc. Pathol. 21(4), 299-306 (2012).

35. Ait-Oufella H, Taleb S, Mallat Z, Tedgui A. Recent advances on the role of cytokines in atherosclerosis. Arterioscler. Thromb. Vasc. Biol. 31(5), 969-979 (2011).

36. Andersson J, Libby P, Hansson GK. Adaptive immunity and atherosclerosis. Clin. Immunol. 134(1), 33-46 (2010).

37. Nakajima N, Nagahiro S, Sano T, Satomi J, Satoh K. Phenotypic modulation of smooth muscle cells in human cerebral aneurysmal walls. Acta Neuropathol. 100(5), 475-480 (2000).

38. Kolega J, Gao L, Mandelbaum M et al. Cellular and molecular responses of the basilar terminus to hemodynamics during intracranial aneurysm initiation in a rabbit model. J. Vasc. Res. 48(5), 429-442 (2011). 
39. Aoki T, Nishimura M, Kataoka H, Ishibashi R, Nozaki K, Hashimoto N. Reactive oxygen species modulate growth of cerebral aneurysms: a study using the free radical scavenger edaravone and $\mathrm{p} 47 \mathrm{phox}^{-/-}$mice. Lab. Invest. 89(7), 730-741 (2009).

40. Aoki T, Kataoka H, Nishimura M, Ishibashi R, Morishita R, Miyamoto S. Ets-1 promotes the progression of cerebral aneurysm by inducing the expression of MCP-1 in vascular smooth muscle cells. Gene Ther. 17(9), 1117-1123 (2010)

41. Hara A, Yoshimi N, Mori H. Evidence for apoptosis in human intracranial aneurysms. Neurol. Res. 20(2), 127-130 (1998).

42. Guo F, Li Z, Song L et al. Increased apoptosis and cysteinyl aspartate specific protease-3 gene expression in human intracranial aneurysm. J. Clin. Neurosci. 14(6), 550-555 (2007).

43. Kanematsu Y, Kanematsu M, Kurihara C et al. Critical roles of macrophages in the formation of intracranial aneurysm. Stroke 42(1), 173-178 (2011).

44. Frosen J, Piippo A, Paetau A et al. Remodeling of saccular cerebral artery aneurysm wall is associated with rupture: histological analysis of 24 unruptured and 42 ruptured cases. Stroke 35(10), 2287-2293 (2004).

45. Aoki T, Kataoka H, Morimoto M, Nozaki K, Hashimoto N. Macrophage-derived matrix metalloproteinase- 2 and -9 promote the progression of cerebral aneurysms in rats. Stroke 38(1), 162-169 (2007).

46. Zhang HF, Zhao MG, Liang GB, Song ZQ, Li ZQ. Expression of pro-inflammatory cytokines and the risk of intracranial aneurysm. Inflammation doi:10.1007/s10753013-9655-6 (2013) (Epub ahead of print).

47. Morgan L, Cooper J, Montgomery H, Kitchen N, Humphries SE. The interleukin-6 gene $-174 \mathrm{G}>\mathrm{C}$ and $-572 \mathrm{G}>\mathrm{C}$ promoter polymorphisms are related to cerebral aneurysms. J. Neurol. Neurosurg. Psychiatry. 77(8), 915-917 (2006).

48. Sun H, Zhang D, Zhao J. The interleukin-6 gene $-572 \mathrm{G}>\mathrm{C}$ promoter polymorphism is related to intracranial aneurysms in Chinese Han nationality. Neurosci. Letters 440(1), 1-3 (2008).

49. McColgan P, Thant KZ, Sharma P. The genetics of sporadic ruptured and unruptured intracranial aneurysms: a genetic meta-analysis of 8 genes and 13 polymorphisms in approximately 20,000 individuals. J. Neurosurg. 112(4), 714-721 (2010).

50. Aoki T, Kataoka H, Shimamura M et al. $\mathrm{NF}-\kappa \mathrm{B}$ is a key mediator of cerebral aneurysm formation. Circulation 116(24), 2830-2840 (2007).

51. Sadamasa N, Nozaki K, Hashimoto N. Disruption of gene for inducible nitric oxide synthase reduces progression of cerebral aneurysms. Stroke 34(12), 2980-2984 (2003).

52. Ishibashi R, Aoki T, Nishimura M, Hashimoto N, Miyamoto S. Contribution of mast cells to cerebral aneurysm formation. Curr. Neurovasc. Res. 7(2), 113-124 (2010).

53. Chyatte D, Bruno G, Desai S, Todor DR. Inflammation and intracranial aneurysms. Neurosurgery 45(5), 1137-1146; discussion 1146-1137 (1999).

54. Hasan D, Chalouhi N, Jabbour P, Hashimoto T. Macrophage imbalance (M1 vs. M2) and upregulation of mast cells in wall of ruptured human cerebral aneurysms: preliminary results. J. Neuroinflamm. 9, 222 (2012).

55. Gui T, Shimokado A, Sun Y, Akasaka T, Muragaki Y. Diverse roles of macrophages in atherosclerosis: from inflammatory biology to biomarker discovery. Mediators Inflamm. 2012, 693083 (2012).

56. Mantovani A, Garlanda C, Locati M. Macrophage diversity and polarization in atherosclerosis: a question of balance. Arterioscler. Thromb. Vasc. Biol. 29(10), 1419-1423 (2009).

57. Passlick B, Flieger D, Ziegler-Heitbrock HW. Identification and characterization of a novel monocyte subpopulation in human peripheral blood. Blood 74(7), 2527-2534 (1989).

58. Tulamo R, Frosen J, Junnikkala $S$ et al. Complement activation associates with saccular cerebral artery aneurysm wall degeneration and rupture. Neurosurgery 59(5), 1069-1076; discussion 1076-1077 (2006).

59. Tulamo R, Frosen J, Paetau A et al. Lack of complement inhibitors in the outer intracranial artery aneurysm wall associates with complement terminal pathway activation. Am. J. Pathol. 177(6), 3224-3232 (2010).

60. Deleo MJ, 3rd, Gounis MJ, Hong B, Ford JC, Wakhloo AK, Bogdanov AA Jr. Carotid artery brain aneurysm model: in vivo molecular enzyme-specific MR imaging of active inflammation in a pilot study. Radiology 252(3), 696-703 (2009).

61. Hasan D, Chalouhi N, Jabbour P et al. Early change in ferumoxytol-enhanced magnetic resonance imaging signal suggests unstable human cerebral aneurysm: a pilot study. Stroke 43(12), 3258-3265 (2012).

62. Hasan DM, Chalouhi N, Jabbour P, Magnotta VA, Kung DK, Young WL. Imaging aspirin effect on macrophages in the wall of human cerebral aneurysms using ferumoxytol-enhanced MRI: preliminary results. J. Neuroradiol. 40(3), 187-191 (2013).

63. Hasan DM, Mahaney KB, Magnotta VA et al. Macrophage imaging within human cerebral aneurysms wall using ferumoxytol-enhanced MRI: a pilot study. Arterioscler. Thromb. Vasc. Biol. 32(4), 1032-1038 (2012).

64. Hasan DM, Mahaney KB, Brown RD Jr et al. Aspirin as a promising agent for decreasing incidence of cerebral aneurysm rupture. Stroke 42(11), 3156-3162 (2011).

65. Aoki T, Nishimura M. The development and the use of experimental animal models to study the underlying mechanisms of CA formation. J. Biomed. Biotechnol. 2011, 535921 (2011).

- Discusses attempts at histopathological reproduction of human cerebral aneurysms in various model organisms and the significance of the derived results.

66. Nuki Y, Tsou TL, Kurihara C, Kanematsu M, Kanematsu Y, Hashimoto T. Elastase-induced intracranial aneurysms in hypertensive mice. Hypertension 54(6), 1337-1344 (2009).

67. Nakashima H, Aoki M, Miyake T et al. Inhibition of experimental abdominal aortic aneurysm in the rat by use of decoy oligodeoxynucleotides suppressing activity of nuclear factor $\kappa \mathrm{B}$ and ets transcription factors. Circulation 109(1), 132-138 (2004).

68. Miyake T, Aoki M, Osako MK, Shimamura M, Nakagami H, Morishita R. Systemic administration of ribbon-type decoy oligodeoxynucleotide against nuclear factor $\mathrm{\kappa B}$ and ets prevents abdominal aortic aneurysm in rat model. Mol. Ther. 19(1), 181-187 (2011).

69. Aoki T, Kataoka H, Ishibashi R, Nozaki K, Hashimoto N. Simvastatin suppresses the progression of experimentally induced cerebral aneurysms in rats. Stroke 39(4), 1276-1285 (2008).

70. Tada Y, Kitazato KT, Yagi K et al. Statins promote the growth of experimentally induced cerebral aneurysms in estrogen-deficient rats. Stroke 42(8), 2286-2293 (2011).

71. Yoshimura Y, Murakami Y, Saitoh M et al. Statin use and risk of cerebral aneurysm rupture: a hospital-based case-control study in Japan. J. Stroke Cerebrovasc. Dis. doi:10.1016/j. jstrokecerebrovasdis.2013.04.022 (2013) (Epub ahead of print).

72. Ni W, Egashira K, Kitamoto S et al. New anti-monocyte chemoattractant protein-1 gene therapy attenuates atherosclerosis in apolipoprotein E-knockout mice. Circulation 103(16), 2096-2101 (2001).

73. Bohley P, Seglen PO. Proteases and proteolysis in the lysosome. Experientia 48(2), 151-157 (1992).

74. Punturieri A, Filippov S, Allen E et al. Regulation of elastinolytic cysteine proteinase 
activity in normal and cathepsin $\kappa$-deficient human macrophages. J. Exp. Med. 192(6), 789-799 (2000).

75. Aoki T, Kataoka H, Ishibashi R, Nozaki K, Hashimoto N. Cathepsin B, K, and S are expressed in cerebral aneurysms and promote the progression of cerebral aneurysms. Stroke 39(9), 2603-2610 (2008).

76. Watanabe T, Morita I, Nishi H, Murota S. Preventive effect of MCI-186 on 15-HPETE induced vascular endothelial cell injury in vitro. Prostaglandins Leukot. Essent. Fatty Acids 33(1), 81-87 (1988).

77. Watanabe T, Yuki S, Egawa M, Nishi H. Protective effects of MCI-186 on cerebral ischemia: possible involvement of free radical scavenging and antioxidant actions. J. Pharmacol. Exp. Ther. 268(3), 1597-1604 (1994).
78. Sanz MJ, Cortijo J, Morcillo EJ. PDE4 inhibitors as new anti-inflammatory drugs: effects on cell trafficking and cell adhesion molecules expression. Pharmacol. Ther. 106(3), 269-297 (2005).

79. Yagi K, Tada Y, Kitazato KT, Tamura T, Satomi J, Nagahiro S. Ibudilast inhibits cerebral aneurysms by down-regulating inflammation-related molecules in the vascular wall of rats. Neurosurgery 66(3), 551-559; discussion 559 (2010).

80. Ishibashi R, Aoki T, Nishimura M, Miyamoto S. Imidapril inhibits cerebral aneurysm formation in an angiotensin-converting enzyme-independent and matrix metalloproteinase-9-dependent manner. Neurosurgery 70(3), 722-730 (2012).

81. Hasan DM, Chalouhi N, Jabbour P et al. Evidence that acetylsalicylic acid attenuates inflammation in the walls of human cerebral aneurysms: preliminary results. J. Am. Heart Assoc. 2(1), e000019 (2013).

- Experimental data suggesting an aspirin regimen may beneficially modulate expression of proinflammatory molecules in the IA wall.

82. Hasan D, Hashimoto T, Kung D, MacDonald RL, Winn HR, Heistad D. Upregulation of cyclooxygenase-2 (COX-2) and microsomal prostaglandin E2 synthase-1 (mPGES-1) in wall of ruptured human cerebral aneurysms: preliminary results. Stroke 43(7), 1964-1967 (2012).

83. Gross BA, Rosalind Lai PM, Frerichs KU, Du R. Aspirin and aneurysmal subarachnoid hemorrhage. World Neurosurg. doi:10.1016/j. wneu.2013.03.072 (2013) (Epub ahead of print). 


\section{Medscape Inflammation and human cerebral aneurysms: current and future treatment prospects}

To obtain credit, you should first read the journal article. After reading the article, you should be able to answer the following, related, multiple-choice questions. To complete the questions (with a minimum $70 \%$ passing score) and earn continuing medical education (CME) credit, please go to www.medscape.org/journal/fnl. Credit cannot be obtained for tests completed on paper, although you may use the worksheet below to keep a record of your answers. You must be a registered user on Medscape.org. If you are not registered on Medscape.org, please click on the New Users: Free Registration link on the left hand side of the website to register. Only one answer is correct for each question. Once you successfully answer all post-test questions you will be able to view and/or print your certificate. For questions regarding the content of this activity, contact the accredited provider,
CME@medscape.net. For technical assistance, contact CME@webmd.net. American Medical Association's Physician's Recognition Award (AMA PRA) credits are accepted in the US as evidence of participation in CME activities. For further information on this award, please refer to http://www.ama-assn.org/ama/pub/ category/2922.html. The AMA has determined that physicians not licensed in the US who participate in this CME activity are eligible for AMA PRA Category 1 Credits $^{\text {TM }}$. Through agreements that the AMA has made with agencies in some countries, AMA PRA credit may be acceptable as evidence of participation in CME activities. If you are not licensed in the US, please complete the questions online, print the AMA PRA CME credit certificate and present it to your national medical association for review.

\section{Activity evaluation: where 1 is strongly disagree and 5 is strongly agree.}

$$
\begin{array}{lllll}
1 & 2 & 3 & 4 & 5
\end{array}
$$

The activity supported the learning objectives.

The material was organized clearly for learning to occur.

The content learned from this activity will impact my practice.

The activity was presented objectively and free of commercial bias.

1. Your patient is a 42-year-old male diagnosed with cerebral aneurysm. Based on the review by Hudson and colleagues, which of the following statements about the role of inflammation in the pathogenesis underlying intracranial aneurysms (IAs) is most likely correct? ?
A Increased hemodynamic stress and atherosclerosis may reduce expression of $E$ and $P$ selectins, monocyte chemotactic protein (MCP)-1, vascular cell adhesion molecule 1 (VCAM-1), and intercellular adhesion molecule-1 (ICAM-1)
B IA pathogenesis requires progressive phenotypic differentiation of vascular smooth muscle cells (VSMCs) to a synthetic and pro-inflammatory state
C Caspase-3, ETS-1, and p47phox are not involved in VSMC phenotypic modulation
D Inflammatory effectors in IA tissue do not include tumor necrosis factor- $\alpha$ (TNF- $\alpha$ ) or interleukin-1 $\beta$ (IL-1 $\beta$ )


2. Which of the following statements about diagnostic imaging of inflammation in IAs is most likely correct??

A Di-5-hydroxytryptamide of gadopentetate dimeglumine is currently approved to image inflammation of IAs in humans

B Ferumoxytol administered to humans with IA allows imaging of inflammation using a special T1*-magnetic resonance imaging (MRI) sequence

C Development of molecular and cellular imaging to monitor improvement or worsening of inflammation in vivo will allow better prediction of the probability of IA rupture

D Early in vivo uptake of ferumoxytol by lymphocytes suggests aneurysmal instability

3. Which of the following statements about potential clinical therapeutic interventions targeting inflammation of IAs is most likely correct? ?

A MMP and MCP-1 stimulation could be a potential target for molecular treatment

B Cathepsin inhibition, free radical scavengers, phosphodiesterase-4 (PDE-4) inhibition, and aspirin administration may help reduce inflammation underlying IAs

$\square$ C NF- $\kappa B$ is not involved in regulation of inflammatory genes during IA formation

D No epidemiological studies support statin use 